\title{
Medidas Preventivas y Consideraciones para la Práctica de Cirugía Oral durante COVID-19
}

\author{
Preventive Measures and Considerations for Oral Surgery Practice During COVID-19
}

\author{
González Quintanilla, David ${ }^{1,3}$ \& Santos-López, Matías ${ }^{2,3}$
}

GONZÁLEZ, Q. D. \& SANTOS-LÓPEZ, M. Medidas preventivas y consideraciones para la práctica de cirugía oral durante COVID-19. Int. J. Odontostomat., 14(3):338-341, 2020.

RESUMEN: EI SARS-CoV-2 es un tipo de coronavirus con un alto grado de contagio entre pacientes y personal de salud, por lo que ha provocado la emergencia sanitaria más grande de los últimos años. El siguiente articulo busca presentar diversas consideraciones para la práctica de la cirugía oral, a fin de disminuir la probabilidad de contagio de SARS-CoV2 en odontólogos. Entre estas destacan, la higiene y lavado de manos tanto de profesionales como de pacientes, medidas de desinfección, bioseguridad y condiciones pre-quirúrgicas, intraoperatorias y postquirúrgicas. Se debe considerar no realizar procedimientos quirúrgicos electivos y en lo posible diferir la atención odontológica en pacientes con padecimientos no urgentes y aquellos con signos y síntomas de COVID-19.

PALABRAS CLAVE: Coronavirus, cirugía oral, COVID-19, SARS- CoV2.

\section{INTRODUCCIÓN}

El 8 de enero del 2020, el Centro Chino para el Control y la Prevención de Enfermedades (CCDC) anunció oficialmente el descubrimiento de un nuevo tipo coronavirus, el COVID-19, considerándolo el responsable de los cuadros de neumonía atípicos que se reportaba en el país desde el mes de diciembre (Li et al., 2020). Con el aumento exponencial de los contagios, el 30 de enero de 2020, la Organización Mundial de la Salud (OMS) declaró al Síndrome Respiratorio Agudo Severo 2 (SARS-CoV-2) y a su enfermedad COVID-19, como una emergencia mundial (Mahase, 2020; Meng et al., 2020). Posteriormente, el 11 de marzo de 2020, lo declaró como una pandemia (Rascado et al., 2020).

La evidencia actual sugiere que el SARS-CoV2 se transmite de persona a persona mediante contacto directo y fluidos respiratorios (Ong et al., 2020). Considerando que el manejo directo de los pacientes sospechosos y contagiados es realizado por el personal de salud, estos se encuentran con un riesgo de contagio extremadamente alto (Meng et al.).
La comprensión del mecanismo de transmisión del SARS-CoV-2, ha llevado a extremar los protocolos de atención en salud, obligando a la desinfección de superficies, uso de antisépticos y uso medidas de protección personal, los cuales permiten el manejo de pacientes sospechosos y contagiados, evitando la propagación viral a través del personal de salud cuando al atención no puede ser diferida (Ren et al., 2020; Honda \& Iwata, 2016).

\section{Consideraciones odontológicas generales}

La práctica odontológica involucra atención directa, generación de aerosoles, contacto con saliva, sangre y otros fluidos corporales. Considerando la transmisión directa de persona a persona, los tiempos de incubación asintomáticos de entre 2 y 10 días, y la fácil propagación del SARS-CoV-2 a través de gotas, manos o superficies contaminadas, la práctica odontológica pasa a ser una actividad con alta exposición al SARS-CoV-2. (Kampf et al., 2020; Peng et al., 2020)

\footnotetext{
${ }^{1}$ Escuela de Odontología, Universidad Autónoma de Chile. Santiago, Chile.

${ }^{2}$ Facultad de Odontología, Universidad Finis Terrae, Santiago, Chile.

${ }^{3}$ Asociación Nacional Científica de Estudiantes de Odontología, Chile.
} 
El síndrome respiratorio agudo severo (SARSCoV), el síndrome respiratorio del Medio Oriente (MERS-CoV) o los coronavirus humanos endémicos (HCoV) pueden persistir en superficies inanimadas como metal, vidrio o plástico hasta 9 días, pero pueden inactivarse eficientemente mediante procedimientos de desinfección de superficie con etanol al $62-71 \%$ previa limpieza con un paño o gasa humedecida con agua o suero fisiológico; peróxido de hidrógeno al 0,5\% durante 1 minuto (Kampf et al.; Fathizadeh et al., 2020) o hipoclorito de sodio al 0,1 - $10 \%$ en periodos de 10 a 30 minutos (Yang et al., 2020).

Los odontólogos y equipo de salud en general, deben tomar medidas estrictas de bioseguridad para la protección de piel y mucosas. Para ello se recomienda la utilización de mascarillas con filtro de partículas FFP2 o tipo N95, guantes de examen, lentes de protección o mascarillas con viseras faciales junto con delantal de aislamiento desechable (American Dental Association, 2020; Meng et al.; Santos-López et al., 2020).

El lavado de manos es considerado uno de los puntos más críticos para reducir la contaminación. Debe ser realizado por el profesional antes y después de cada atención, así como también por los pacientes, quienes al menos deben higienizar sus manos con alcohol gel o similar, previo y posterior a la atención, además, el doble enguantado y la desinfección de estos guantes una vez puestos y al momento de terminar el procedimiento, sería una medida positiva para el control de la propagación de virus y bacterias (Verbeek et al., 2020).

Previo a la atención odontológica o posterior al examen clínico si este es necesario, se recomienda una adecuada categorización y decisión de la necesidad de atención del paciente, evaluando la posibilidad de diferir el procedimiento si este es de carácter electivo (Araya, 2020).

\section{Consideraciones previas al procedimiento quirúrgico}

En la evaluación de pacientes durante el brote de COVID-19, se recomienda que las clínicas dentales establezcan triages previos al control, para medir y registrar la temperatura de cada personal y paciente como un procedimiento de rutina. El personal debe hacer preguntas a los pacientes sobre el estado de salud y el historial de contacto o viaje (American Dental Association).
La utilización de enjuagues antisépticos previo a la atención odontológica favorecería la disminución del virus en la cavidad oral. La elección del antiséptico debe ser basada en permitir una adecuada disminución de la carga salival, sin generar daño en la mucosa oral (Meng et al). EI SARS-CoV-2 es vulnerable a la oxidación, por lo cual, el peróxido de hidrógeno diluido al $1 \%$ sería efectivo como antiséptico (American Dental Association; Peng et al.; Kampf et al.). Para obtener $15 \mathrm{~mL}$ de enjuague en una concentración próxima al $1 \%$, se puede utilizar $5 \mathrm{~mL}$ de peróxido de hidrogeno $10 \mathrm{Vol}$. adicionando $10 \mathrm{~mL}$ de agua destilada (Araya). También se ha descrito que la utilización de povidona yodada (PVP-I) en concentraciones de $0,23 \%$ a $7 \%$, demuestra una actividad virucida altamente efectiva contra una amplia gama de virus, incluidos varios coronavirus responsables de epidemias recientes, como SARS-CoV y MERS-CoV (Parhar et al., 2020).

Respecto al uso de exámenes complementarios, se debe preferir el uso de exámenes radiográficos extraorales a fin de disminuir el contacto con la saliva, como: radiografía panorámica con utilización protector de plástico desechable para posicionador o tomografía computada o de haz cónico, donde no existe contacto de saliva con la máquina. Se debe considerar el costo para el paciente, el rendimiento del examen y requerimientos del clínico (Meng et al.; Araya).

Se debe considerar la utilización de anestesia troncular, más complemento infiltrativo en primera instancia, evitando posibles refuerzos anestésicos durante el procedimiento y la disminución de insumos eliminados posteriormente que podrían estar contaminados (Zhao \& Gao, 2020; Ellis et al., 2020).

Además, considerar la hidratación previa del personal, antes de posicionar los elementos de protección personal, para disminuir las pausas innecesarias y las probabilidades de error durante el procedimiento quirúrgico (Ellis et al.).

\section{Consideraciones durante el procedimiento quirúrgico}

Para procedimientos quirúrgicos orales, se ha descrito que la atención ideal se debe llevar a cabo en salas de procedimiento con presión negativa como pabellones, reduciendo al mínimo el personal presente (Meng et al.), siguiendo las técnicas quirúrgicas según requiera el diente o zona a tratar. Si el procedimiento o la condición del paciente amerita indicación 
de sutura, se debe dar preferencia materiales reabsorbibles con el propósito de evitar retirarla en procedimientos posteriores (Meng et al.).

Tanto para la irrigación de alveolos dentales, así como para el uso de elementos rotatorios de baja velocidad, es aconsejable la aspiración de alta potencia, se puede además poner la mano libre del operador cubriendo parcialmente la boca del paciente para disminuir la generación de aerosoles y gotas mientras se utiliza el equipo de protección personal (Meng et al.; Peng et al.; Araya).

\section{Consideraciones post procedimiento quirúrgico}

Una vez terminado el procedimiento se deben desinfectar los elementos de protección personal utilizados antes de su eliminación y superficies comprometidas con etanol al 62-71\% previa limpieza con un paño o gasa humedecida con agua, suero fisiológico, peróxido de hidrógeno al $0,5 \%$ durante 1 minuto (Kampf et al.; Fathizadeh et al.) o hipoclorito de sodio al $0,1-10 \%$ en periodos de 10 a 30 minutos (Yang et al.).

La prescripción de analgésicos y antiinflamatorios no esteroideales AINEs de uso común en Odontología se podría ver afectada. El comité de evaluación de riesgos de farmacovigilancia de la Agencia Europea de Medicamentos (PRAC) ha recomendado no utilizar medicamentos que contienen ibuprofeno y ketoprofeno para advertir que pueden enmascarar los síntomas de infección (Torjesen, 2020a). No obstante, actualmente se señala que no hay evidencia suficiente para establecer un vínculo entre el uso de ibuprofeno $u$ otros AINEs y contracción o empeoramiento del COVID-19. La Agencia Reguladora de Medicamentos y Productos Sanitarios (MHRA), NHS Inglaterra y el Instituto Nacional de Excelencia en Salud y Atención (NICE) ha actualizado sus consejos para decir que los pacientes pueden tomar paracetamol o ibuprofeno para síntomas de COVID-19 (Torjesen, 2020b). Para efectos de esta revisión, se sugiere no prescribir fármacos que contengan en sus formulaciones ibuprofeno y ketoprofeno.

Además, se sugiere realizar un seguimiento efectivo a todo paciente por un periodo de 8 a 14 días, para visualizar complicaciones, realizar controles excepcionales no postergables y definir acciones en caso de diagnóstico de COVID-19 posterior a la atención odontológica (Hua et al., 2020).

\section{CONCLUSIONES}

La práctica odontológica genera una alta exposición al SARS-CoV-2 y por lo tanto, riesgo de contagio de COVID-19. La cirugía bucal en pacientes con patologías no urgentes, debe ser diferida a fin de disminuir contagios y exposición al SARS-CoV-2. En caso de tratamientos quirúrgicos impostergables, es fundamental la anamnesis y evaluación de síntomas. Al indicarse la atención, se deben seguir los protocolos previos de desinfección, protección personal, exámenes complementarios y ergonomía. Durante la atención, los protocolos para disminución de aerosoles, y posterior a la atención, protocolos de seguimiento tanto del procedimiento como de alguna posible sintomatología infecciosa.

GONZÁLEZ, Q. D. \& SANTOS-LÓPEZ, M. Preventive measures and considerations for oral surgery practice and during COVID-19. Int. J. Odontostomat., 14(3):338-341, 2020.

ABSTRACT: SARS-CoV-2 is a type of coronavirus with a high degree of contagion between patients and health personnel, which is why it has caused the largest health emergency in recent years. The following article seeks to present various recommendations for the practice of oral surgery in order to reduce the probability of contagion of SARSCoV-2 for Dental Surgeons. The most outstanding are hygiene and hand washing for both professionals and patients, disinfection measures, biosecurity and pre-surgical, intraoperative and post-surgical conditions. Consideration should be given not to perform elective surgical procedures and, where possible, defer dental care in patients with non-urgent conditions and those with signs and symptoms of COVID-19.

KEY WORDS: Coronavirus, oral surgery, COVID19, SARS-CoV-2.

\section{REFERENCIAS BIBLIOGRÁFICAS}

American Dental Association. Coronavirus Frequently Asked Questions. ADA Center for Professional Success. Sitio Web. American Dental Association, 2020. Disponible en: https:// success.ada.org/en/practice-management/patients/coronavirusfrequently-asked-questions

Araya, S. C. Consideraciones para la atención de urgencia odontológica y medidas preventivas para COVID-19 (SARS-CoV 2). Int. J. Odontostomat., 14(3):268-70, 2020

Ellis, R.; Hay-David, A. G. C. \& Brennan, P. A. Operating during the COVID-19 pandemic: How to reduce medical error. Br. J. Oral Maxillofac. Surg., 2020. DOI: https://www.doi.10.1016/ j.bjoms.2020.04.002 
Fathizadeh, H.; Maroufi, P.; Momen-Heravi, M.; Dao, S.; Köse, S.; Ganbarov, K.; Pagliano, P.; Esposito, S. \& Kafil, H. S. Protection and disinfection policies against SARS-CoV-2 (COVID-19). Infez Med., 28(2):185-91, 2020.

Honda, H. \& Iwata, K. Personal protective equipment and improving compliance among healthcare workers in high-risk settings. Curr. Opin. Infect. Dis., 29(4):400-6, 2016.

Hua, C. G.; Liu, Z. Q.; Wang, Q.; Yang, Z.; Xu, Q. H. \& Zhang, J. Strategy of dental clinics to cope with the epidemic period of infectious diseases based on the experience of corona virus disease outbreak. Hua Xi Kou Qiang Yi Xue Za Zhi, 38(2):117121, 2020.

Kampf, G.; Todt, D.; Pfaender, S. \& Steinmann, E. Persistence of coronaviruses on inanimate surfaces and their inactivation with biocidal agents. J. Hosp. Infect., 104(3):246-51, 2020. Disponible en: https://www.journalofhospitalinfection.com/article/S01956701(20)30046-3/fulltext

Li, Q.; Guan, X.; Wu, P.; Wang, X.; Zhou, L.; Tong, Y.; Ren, R.; Leung, K. S. M.; Lau, E. H. Y.; Wong, J. Y.; et al. Early transmission dynamics in Wuhan, China, of novel coronavirus- infected pneumonia. N. Eng. J. Med., 382(13):1199-207, 2020.

Mahase, E. China coronavirus: WHO declares international emergency as death toll exceeds 200. BMJ, 368:m408, 2020.

Meng, L.; Hua, F. \& Bian, Z. Coronavirus disease 2019 (COVID- 19): emerging and future challenges for dental and oral medi- cine. J. Dent. Res., 2020. DOI: https://www.doi.org/10.1177/ 0022034520914246

Ong, S. W. X.; Tan, Y. K.; Chia, P. Y.; Lee, T. H.; Ng, O. T.; Wong, M. S. Y. \& Marimuthu, K. Air, Surface Environmental, and Personal Protective Equipment Contamination by Severe Acute Respiratory Syndrome Coronavirus 2 (SARS-CoV-2) From a Symptomatic Patient. JAMA, 2020. DOI: https://doi.org/ 10.1001/jama.2020.3227

Parhar, H. S.; Tasche, K.; Brody, R. M.; Weinstein, G. S.; O'Malley, B. W. Jr.; Shanti, RM, Newman JG. Personal protective equipment for preventing highly infectious diseases due to exposure to contaminated body fluids in healthcare staff. Head Neck, 2020 Apr 25. doi:10.1002/hed.26200

Peng, X.; Xu, X.; Li, Y.; Cheng, L.; Zhou, X. \& Ren, B. Transmission routes of 2019-nCoV and controls in dental practice. Int. J. Oral Sci., 12(1):9, 2020. Disponible en: http://www.nature.com/articles/ s41368-020-0075-9

Ren, Y. F.; Rasubala, L.; Malmstrom, H. \& Eliav, E. Dental Care and Oral Health under the Clouds of COVID-19. JDR Clin. Trans. Res., 2020. DOI:https//www.do.org/10.1177/2380084420924385.

Santos-López, M.; Jaque-Ulloa, D. \& Serrano-Aliste, S. Decontamination methods and reuse of filtering facepiece respirators during SARS-CoV-2 outbreak. Int. J. Odontostomat., 14(3):310-5, 2020.

Torjesen, I. Covid-19: ibuprofen can be used for symptoms, says UK agency, but reasons for change in advice are unclear. BMJ. 2020a. DOI:https//www.doi.org/10.1136/bmj.m1555

Torjesen, I. Ibuprofen can mask symptoms of infection and might worsen outcomes, says European drugs agency. BMJ. 2020b DOI:htpps//www.doi.org/10.1136/bmj.m1614

Verbeek, J. H.; Rajamaki, B.; ljaz, S.; Sauni, R.; Toomey, E.; Blackwood, B.; Tikka, C.; Ruotsalainen, J. H. \& Kilinc Balci, F. S. Personal protective equipment for preventing highly infectious diseases due to exposure to contaminated body fluids in healthcare staff. Cochrane Database Syst. Rev. 4:CD011621, doi: 10.1002/14651858.CD011621.pub4.

Yang, Y.; Soh, H. Y.; Cai, Z. G.; Peng, X.; Zhang, Y. \& Guo, C. B. Experience of Diagnosing and Managing Patients in Oral Maxillofacial Surgery during the Prevention and Control Period of the New Coronavirus Pneumonia. Chin. J. Dent. Res., 2020;23(1):57-62. DOI: htpps://www.doi.org 10.3290/ j.cjdr.a44339.
Zhao, Z. \& Gao, D. Precaution of 2019 novel coronavirus infection in department of oral and maxillofacial surgery. $\mathrm{Br}$. J. Oral Maxillofac. Surg. 58(3):250-3, 2020.

Dirección para correspondencia

David González Quintanilla

Escuela de Odontología

Universidad Autónoma de Chile

Santiago

CHILE

Email: davidgonzalez.odonto@gmail.com

Recibido : 28-04-2020

Aceptado: 29-04-2020 